\title{
Impact of simultaneous pancreas-kidney transplantation: patients' perspectives
}

\author{
This article was published in the following Dove Press journal: \\ Patient Preference and Adherence \\ 22 August 2012 \\ Number of times this article has been viewed
}

\author{
P Isla Pera' \\ J Moncho Vasallo² \\ O Guasch Andreu ${ }^{3}$ \\ MJ Ricart Brulles ${ }^{4}$ \\ A Torras Rabasa ${ }^{4,5}$ \\ 'Public Health Department, Nursing \\ School, Universitat de Barcelona, \\ Barcelona, ${ }^{2}$ Community Nursing, \\ Preventive Medicine and Public Health \\ and History of Science, Universidad \\ Alicante, ${ }^{3}$ Facultat Sociologia, \\ Universitat de Barcelona, ${ }^{4}$ Institut \\ Clínic de Nefrología i Urología, \\ Hospital Clínic de Barcelona, ${ }^{5} \mathrm{Facultat}$ \\ de Medicina, Universitat de Barcelona, \\ Barcelona, Spain
}

Correspondence: Pilar Isla Pera

Campus Universitari Ciències de la Salut,

Universitat Barcelona, Feixa Llarga s/n

L'Hospitalet de Llobregat 08907, Spain

$\mathrm{Tel}+34934024241$

Fax +34934024297

Email pisla@ub.edu
Background: Few qualitative studies of simultaneous pancreas-kidney transplantation (SPK Tx) have been published. The aims of this study were to explore from the perspective of patients, the experience of living with diabetes mellitus type 1 (T1DM), suffering from complications, and undergoing SPK Tx with good outcome; and to determine the impact of SPK Tx on patients and their social and cultural environment.

Methods: We performed a focused ethnographic study. Twenty patients were interviewed. Data were analyzed using content analysis and constant comparison following the method proposed by Miles and Huberman.

Results: A functioning SPK Tx allowed renal replacement therapy and insulin to be discontinued. To describe their new situation, patients used words and phrases such as "miracle", "being reborn" or "coming back to life". Although the complications of T1DM, its surgery and treatment, and associated psychological problems did not disappear after SPK Tx, these were minimized when compared with the pretransplantation situation.

Conclusion: For patients, SPK Tx represents a recovery of their health and autonomy despite remaining problems associated with the complications of T1DM and SPK Tx. The understanding of patients' existential framework and their experience of disease are key factors for planning new intervention and improvement strategies.

Keywords: qualitative research, ethnography, complications of diabetes mellitus type 1, simultaneous pancreas-kidney transplantation

\section{Introduction}

The outcome of simultaneous pancreas-kidney transplantation (SPK Tx) in patients with type 1 diabetes mellitus (T1DM) has dramatically improved in recent years due to optimized surgical techniques and new immunosuppressive drug regimens. Normoglycemia is followed by stabilization or even regression of diabetic lesions. ${ }^{1}$ Nevertheless, to a greater or lesser degree, complications and inconveniences (physical and psychological alterations, secondary effects of immunosuppressive therapy and the need for lifelong medication and medical follow-up) can exist after SPK Tx..$^{2-6}$ Many studies have shown the effectiveness of SPK Tx in improving health-related quality of life (HR-QoL). ${ }^{2,7-9}$

However, HR-QoL questionnaires have certain limitations. Criticisms have focused on methodological difficulties and the consequent biases that can be produced by measuring HR-QoL, ${ }^{5,10}$ the absence of specific definitions of this concept in various studies, and the lack of attention paid to patients' feelings. ${ }^{6,10}$ No single measure can 
suit every purpose or application but, when measures are selected inappropriately and data misinterpreted, any conclusions drawn are fundamentally flawed.

In the last few years, interest has grown in identifying the subjective perception of transplant recipients through qualitative studies without the use of psychometric instruments or standardized tests which, in addition to the abovementioned problems, limit information collection by evaluating only predefined variables.

Few qualitative studies of SPK Tx have been published. ${ }^{11}$ Such studies are of interest because qualitative methodology is well suited to describe and understand the complex and multidimensional reality of having T1DM, suffering from severe complications, and undergoing SPK Tx. Consequently, after studying HR-QoL in patients with SPK Tx² we elected to perform an ethnographic study in patients with SPK Tx who had both grafts functioning. The aims of this study were to explore from the perspective of patients, the experience of living with T1DM, suffering from complications, and undergoing SPK Tx with good outcome; and to determine the impact of SPK Tx on patients and their social and cultural environment.

\section{Patients and methods}

An ethnographic method ${ }^{12}$ and symbolic interactionism ${ }^{13}$ were used. Ethnography, embedded in an anthropological tradition, is essentially the study of a particular cultural group or phenomenon. Ethnographers use observation and interviews to help classify and organize an individual's perception of reality. ${ }^{14}$ In our study, participants consisted of 20 patients who underwent SPK Tx in Barcelona (Spain) from 1998-2002. Data were collected from 2004-2006. Intentional sampling was performed and was representative of all groups of the patient population (age, sex, year of SPK Tx, and geographical origin). The criterion of data saturation was used to establish the number of participants. ${ }^{15}$ Saturation was apparent when no new information was discovered during analysis of the interview. ${ }^{15}$ Participant observation was carried out in the waiting rooms of medical consultations for 2 months in order to observe the patients' relationships with family and healthcare professionals.

The criterion for inclusion of patients was the continued functioning of both grafts. The first author conducted all interviews in a private office of a hospital. The interviews lasted between 70 and 90 minutes and were audio recorded. The interview format was nondirective. Questions were openended and broad, and were presented in a flexible manner.
The interviewer made an effort to acknowledge her assumptions about the phenomenon under study and to take into account the potential impact of her subjectivity on the data. These strategies were used in an effort to reduce bias, and to gain a thorough understanding of the phenomenon under study that was as representative as possible of the participants' perspectives. ${ }^{16}$ Data were analyzed with the software tool Atlas T (Atlas.ti Scientific Software Development, Berlin, Germany) using content analysis, and constant comparison following the method proposed by Miles and Huberman ${ }^{15}$ consisting of data reduction, data display, and conclusion drawing/verification. To improve the study's validity, the information obtained was fed back to the informants at the end of the interview. Data analysis was performed independently by two researchers and was discussed with the rest of the team. As complementary variables, we recorded age, sex, T1DM duration, and year of SPK Tx in patients. Permission to perform the research was obtained from the ethics committee of the hospital. Each participant was informed of the purpose of the research and signed a consent form guaranteeing patient anonymity and confidentiality.

\section{Results}

Patients' sociodemographic data are shown in Table 1.

The themes identified from analysis of the interviews were grouped into two stages: before SPK Tx and after SPK Tx. In the first stage, two themes were identified: the loss of integrity and feeling guilty and alone; and being a candidate for SPK Tx. In the second stage, four themes were identified: transplantation or living a miracle; enjoying what is important; the gift of life with limitations; and the spiritual dimension.

Table I Informants' (patients) sociodemographic data

\begin{tabular}{llll}
\hline & Men & Women & Total \\
\hline Sex & $8(40 \%)$ & $12(60 \%)$ & - \\
Mean age & $45.7 \pm 7.8$ & $38.3 \pm 5.9$ & - \\
Mean TIDM duration & $27.5 \pm 13$ & $24.3 \pm 10.4$ & - \\
$\begin{array}{l}\text { Geographical origin } \\
\quad \text { Catalonia }\end{array}$ & - & & \\
$\quad$ Other region of Spain & - & - & $10(50 \%)$ \\
Year of SPK Tx & & & $10(50 \%)$ \\
1998 & 2 & 2 & - \\
1999 & 2 & 3 & - \\
2000 & 1 & 3 & - \\
2001 & 1 & 2 & - \\
2002 & 2 & 2 & - \\
\hline
\end{tabular}

Abbreviations: TIDM, diabetes mellitus type I; SPK Tx, simultaneous pancreas-kidney transplantation. 


\section{Before SPK Tx}

\section{Loss of integrity and feeling guilty and alone}

Patients and their relatives depicted T1DM as a destructive disease that was difficult to control and to live with. They described the onset and progression of complications and the changes produced in their personal and family lives with emotion. Because of the association between the development of complications and poor metabolic control, patients felt guilty and blamed. The blame culture, where the disease is perceived as divine punishment was replaced by the culture of shame that associates the disease with weakness and self-neglect. ${ }^{17}$ The start of renal replacement therapy provoked fear and anxiety. Catheter placement and the need to depend not only on insulin but also on a machine to stay alive, was interpreted as an assault against bodily integrity, and contributed to poorer self-image and increased stigma: "Insulin and dialysis is the worst thing that can happen to you. It changes your metabolism, you lose muscle, your legs become weak [...], everything changes," (27-year-old man). Problems with erection and ejaculation were associated with sexual impotence or lack of sexual desire and limited the possibility of having children, and this led some men to question their masculinity. Physical and psychological limitations interfered with work and leisure activities. Friends stopped calling: "Our colleagues, friends, stopped calling. We didn't phone anybody either. What could we tell them?" (36-year-old woman).

\section{Being a candidate for SPK Tx}

Patients described the news that a transplant was available as an event that was both hopeful and stressful, generating emotional conflicts. For patients, SPK Tx represented the recovery of "health" and freedom from suffering and the need to depend on a machine or on insulin to stay alive, but at the same time, they feared the intervention and possible graft rejection: "When they told me about the transplant, I got lots of encouraging messages to keep my morale high but you don't really worry that much. I was very afraid but I wanted it done because I wanted to see my children grow up" (38-year-old man).

When faced with SPK Tx, patients reviewed their priorities and for most, the risk of death was the lesser of two evils: "Whatever happens, things will be much better than what they are now" (45-year-old woman). The time spent on the waiting list provoked anxiety and also, guilt about waiting expectantly for the telephone call and about wanting the waiting time to end as soon as possible (which implicitly represented waiting for the death of a donor):

I always had my ear to the telephone. And when it rang!

Especially when it rang at night because almost everyone is rung at night. Some nights I would wake and I didn't know if it had rung and I'd ask my wife "Has the telephone rung?" You're selfish but you want the waiting to be over right now. [38-year-old man]

Waiting for and wanting the telephone call that would confirm the existence of a compatible donor did not prevent the occurrence of nervousness and contradictory feelings in patients when the telephone call came. The strongest emotion expressed by patients was a psychological "block" that prevented them from thinking about the possible effects of the intervention and the transplant: "When they rang me, I don't know what I felt. I didn't feel anything, neither good nor bad. It's something you don't want to think about" (45-year-old woman).

\section{After SPK Tx}

\section{Transplantation or "living a miracle"}

To describe their new situation, interviewees used words such as "miracle" or "being reborn". Patients reported that they had not only regained their health, but also their skin color, the gleam in their eyes, vitality, social relationships and, in some cases, sexual and reproductive function.

After SPK Tx, recipients had the perception they were enjoying normal life, something that they had not done since the diagnosis of T1DM. The end of dependence on dialysis and insulin, and the end of hypoglycemic episodes were interpreted as recovering health and autonomy. Recipients expressed their happiness at not having to depend on their principal care provider, at being able to be alone, travel, to (in some cases) return to work, and especially, recover sexual and reproductive ability: "Now, thanks to the transplant, I'm expecting a child. It's a boy and I get excited just talking about it" (40-year-old woman).

\section{Enjoying "what is important"}

SPK Tx led to a restructuring of patients' experience. Before the intervention, patients conquered their beliefs and fear of the operation by reevaluating their values, and after SPK, they were able to value and prioritize those things that were essential to their happiness: "You change the way you think. For me, the most important thing is the family's wellbeing, and enjoying seeing the sunrise" (53-year-old man). 
There was also a change in the way recipients experienced time: "Before I let time pass. Now I think time flies and I have to enjoy every moment of life" (36-year-old woman).

\section{A "gift of life" with limitations}

Despite the value placed on SPK Tx by patients, they also reported various types of problems, grouped into the following subcategories.

\section{Physical problems and effects of treatment}

After SPK Tx, the perception of improvement was undeniable but most recipients reported physical problems due to the complications of T1DM, surgery, and immunosuppressive treatment: "Now my sight is worse than ever, but just thinking that I don't inject insulin or have hypoglycemic episodes is enough to make me happy" (36-year-old woman). Immunosuppressive agents were described as strong drugs with annoying effects that could cause severe health problems. The effects differed between men and women. Men reported abdominal distension and gastrointestinal problems while women reported increased hair growth and sweating. However, the benefits of SPK Tx were so great, and the fear of rejection so strong, that patients played down the possible risks of immunosuppressive treatment.

\section{Fear of graft loss}

For patients, SPK Tx represented the only option to achieve a healthy life but was threatened, because the organs were foreign and could be rejected. Recipients coped with this differently, with some trying to live fully in the present: "I don't want to think about possible problems because I want to live in the present" (45-year-old woman). Others expressed fear, but also hope about scientific advances: "I have faith in the future. If this fails, I know that they will take organs from embryos and, if not, there are pig organs" (36-year-old woman). In yet other patients, the perception of threat started at surgery and was constant: "I was very sad after the operation and others have felt the same because you think 'All this for nothing if I lose it!' And later, you're always thinking 'How long can it last?"' (36-year-old woman).

\section{Problems in reconstructing identity}

Organ transplants are an addition to biological identity, and can be difficult to assimilate. The recipients differed in their attitudes to the organs and donors. Some saw the transplanted organs as if they were their own: "Thanks to him, I am the way I am, but I also think that now the kidney and pancreas are mine. We should all donate organs" (29-year-old woman). Other recipients adopted an intermediate position. The organs were theirs but were also the donor's and they felt indebted to the donor, and to his or her family: "The kidney and pancreas belong to a woman who died and are also mine. There's not a single moment when I don't remember her and her family" (38-year-old man). In other cases, identification with the donor went further. Some patients corporealized the donor, who appeared in their dreams, while others even gave the donor a name, and integrated the imaginary figure of the donor in their daily routine and family group: "I dream about the donor, although I don't know if it's really him or if it's how I imagine him" (53-year-old man); and "One thinks a lot about the donor because the pancreas and kidney are his. My mother and I call him Jesús. We speak about him a lot and say Mass for him" (36-year-old woman).

An obsession with the donor and imaginary ideas about what the donor was like, when and how he or she would die (if still alive), and how the identity of the donor would affect the recipient's own identity, started before the intervention and affected the interviewees to a greater or lesser extent. However, sometimes there were beliefs and myths that generated intense dread: "Of course I wanted the transplant but I was afraid! I thought that afterward, when I was asleep, he would probably appear and touch me" (50-year-old woman). The secrecy surrounding information on donation inhibited patients and their families from talking with the medical team about this type of problem. Health professionals also avoided asking about these topics, which were consequently relegated to the personal or familial sphere.

\section{Need for medical follow-up and treatment}

After SPK Tx, patients must continue treatment and follow-up for the rest of their lives. All recipients reported that they complied with drug treatment, tried to follow a healthy life style, and attended medical appointments without opposition. These patients had successful experiences of the most advanced biotechnology, believed in medical science, became dependent on their doctors and were undemanding. One form of reciprocity with health professionals was the expression of gratitude and acceptance of recommendations without argument: "I've come here whenever they've told me to and I don't mind if I have to wait 2 hours or 4 [hours]" (40-year-old woman). These attitudes did not prevent some recipients from criticizing the health system or some health professionals, but none of the complaints concerned the Transplant Unit. 


\section{Spiritual dimension}

All the interviewees reported that their religious beliefs were unchanged after SPK Tx. Most described themselves as lapsed or semi-lapsed Catholics. However, suffering and pain had moral consequences even among the nonreligious. The question "What have I done to deserve this?" was common among interviewees. One elderly family member believed in the power of prayer but most interviewees rejected or rebelled against religion, and placed their faith in science: "When they operated on me, my mother said 'Thank God that the operation has gone well' and my daughter told her 'Don't thank God, thank the doctors"' (53-year-old man).

\section{Discussion}

In childhood or adolescence, T1DM is incorporated into the identity of affected individuals, leading to stigma and to the disease becoming inseparable from their life stories. Therefore the experience of SPK Tx cannot be studied in isolation and must be interpreted within the context of the disease. End stage renal disease and renal replacement therapy increased the perception of loss of integrity and functionality and affected social relationships. This finding is in agreement with those of other studies reporting an association between renal replacement therapy and increased stress and anxiety and impaired QoL, ${ }^{18-20}$ as well as a close relationship between QoL and morbidity and mortality. ${ }^{21}$

Currently, organ donation and transplantation are routine practice in many hospitals but profound social and ideological changes have had to take place for this to occur. The concepts of "brain death" and "useful death" are rationalized concepts accepted by the scientific community, and by some patients and their relatives but for others, organ transplantation challenges historically elaborated beliefs and perceptions about the meaning of life, and the sense of transcendence beyond death. For some patients, transplanted organs are an addition to biological identity that is difficult to assimilate, as they represent not only the physical but also the imaginary and symbolic incorporation of the donor's organs. ${ }^{22}$ SPK Tx recipients live with two cadaveric organs that can be from a person differing in sex and age from the recipient, and some recipients show psychological disorders that include anxiety and identification with the donor and the donor's family. These fantasies could be interpreted as useful, specific-adaptation strategies after transplantation ${ }^{11}$ however, the present study found that these strategies occurred independently of the time since SPK Tx, and other studies have described similar problems. ${ }^{23,24}$ The Frankenstein syndrome held by some patients, consists of the perception of being reconstituted from bits and pieces of cadavers, and the sensation of living with a foreign body part that introduces some of its features into the recipient's identity. ${ }^{25}$

Other psychological disorders have also been described after transplantation, such as sexual dysfunction, ${ }^{3}$ difficulties in adapting, ${ }^{4,5}$ dissatisfaction with body image, ${ }^{6}$ and problems with treatment adherence. ${ }^{7,26,27}$ In this study, the patients reported that their sexual function had improved, and that they complied with drug treatment and maintained a healthy lifestyle. Difficulties in adapting and changes in body image were accepted as the price that had to be paid for the benefits obtained. Poor treatment adherence has previously been related to family support, ${ }^{23-28}$ to the coping strategies acquired to reduce stress and adapt to the disease,${ }^{27}$ and to pre- and posttransplantation expectations. ${ }^{23,29}$ In this study, the patients had family support and positive expectations of SPK Tx, believing that nothing could be worse than a future without SPK Tx, and had acquired coping strategies that allowed them to adapt to the changes, actively alternating between strength and feeling discouraged. This difference with patients undergoing other types of transplantation can be explained by their youth, long disease duration, family support, and a prolonged health professional-user relationship, aiding knowledge and experience. The difference in the adverse effects of immunosuppressive treatment perceived by men and women coincides with the findings of other studies, ${ }^{30}$ and could be due to the higher value placed by women on effects related to body image.

In the patients' narratives, expressions of discouragement and despair alternated with those of hope for a better future. Ambiguity, due to the conflict between scientific and supernatural thought, has been reported in other studies. ${ }^{31}$ Patients in our study, however, expected miracles from science and had little faith in the power of religion or magic, unlike those from other cultures, who showed a greater religiosity, favoring the acceptance of a divine scheme and hope regarding the progress of the disease. ${ }^{31,32}$

The adventure of SPK Tx is like a rite of passage, an initiation in which the individual is not and never will be, the same as before receiving the transplant. After years of illness, complications and disability, a surgical procedure implants a new kidney and pancreas in the recipient's body. If the procedure is successful, end stage renal disease will be cured, patients will cease to be diabetic, and will experience physical, psychological, and social changes that will transform their lives as well as their philosophy of life.

In conclusion, SPK Tx improves the perception of quality of life and of freedom to the extent that patients no 
longer feel ill. This finding is in agreement with some quality of life studies reporting similar, or even superior results to those in the healthy reference population, in some health dimensions..$^{2,8,9}$ However, as with the study by Ross et al, ${ }^{33}$ we found a discrepancy between expressions such as "new life" and "feeling well" and patients' reports of physical and psychological problems of distinct intensity, which could be treated if recognized and evaluated by health professionals. HR-QoL questionnaires have limitations, such as their lack of sufficient flexibility to adjust to the specific context in which patients live and cope with their disease, and the difficulty of identifying and understanding complex physical, psychological, and functional variables through a simple numerical evaluation. Some authors have modified and validated scales of quality of life following a review of the literature and a series of interviews with patients. ${ }^{34}$

The conclusions that can be drawn from the results of this study are limited by the following factors. The strategy adopted for data collection may have been less than optimal since the interview did not encourage open discussion (as would have occurred in a focus group) of the topic studied, since half of the patients and family members interviewed did not live in Catalonia, and the interviews had to take place on the day of the medical appointment to avoid travelling by patients, caregivers, and researchers. Also, the time from SPK Tx ranged from 4-6 years but it would have been interesting to interview the participants from an earlier point in time to capture their experience prospectively.

\section{Conclusion}

The end of dependence on dialysis and insulin, and the end of hypoglycemic episodes were interpreted as the recovery of health and autonomy, but our results suggest that SPK Tx recipients require not only medical monitoring, but also psychological support before, and after transplantation. A psychosocial evaluation is necessary to ensure high-quality care.

Despite the efforts of some health professionals and the theoretical development of the healthcare model, the framework that currently predominates continues to be the biomedical model, which does not sufficiently contemplate the complexity of patients' and their families' experiences, which include psychological and sociocultural features as well as organic and functional aspects.

Future studies should perform temporal evaluations to determine the variations produced after Tx SPK and should combine quantitative and qualitative methods to provide more exhaustive information on this topic. HR-QOL instruments only evaluate the dimensions that patients experience directly and exclude other biological and socio-environmental variables that also affect their health. Qualitative investigation allows researchers to gain access to the world of emotions, feelings, and daily experiences, and insight into the impact of advanced technologies on patients, their facilities, and the social milieu of the individuals involved; this type of research also aids reflection on the social role of health professionals' practice.

\section{Authors' contributions}

Pilar Isla Pera: the main researcher. Conceived and designed the study, prepared the manuscript, and participated in data collection and results analysis and discussion and final review of the manuscript.

Joaquin Moncho-Vasallo: participated in data collection, results analysis, and discussion and final review of the manuscript.

Oscar Guasch-Andreu: participated in data collection, results analysis, and discussion and final review of the manuscript.

María José Ricart Brulle: participated in data collection, and discussion and final review of the manuscript.

Alberto Torras-Rabasa: participated in data collection, results analysis, and discussion and final review of the manuscript.

\section{Disclosure}

This study was supported by the Fondo de Investigaciones Sanitarias (2004-2006) Ministry of Health and Consumer Affairs, Spain (grant number P1041210) to Pilar Isla.

The authors declare they have no conflicts of interest.

\section{References}

1. Morath C, Schmied B, Mehrabi A, et al. Simultaneous pancreaskidney transplantation in type 1 diabetes. Clin Transplant. 2009; 23 Suppl 21:S115-S120.

2. Isla Pera P, Moncho Vasallo J, Torras Rabasa A, et al. Quality of life in simultaneous pancreas-kidney transplant recipients. Clin Transplant. 2009;23(5):600-605.

3. Trumper A, Appleby L. Psychiatric morbidity in patients undergoing heart, heart and lung, or lung transplantation. J Psychosom Res. 2001;50(2):103-105.

4. Kita Y, Fukunishi I, Harihara Y, et al. Psychiatric disorders in living-related liver transplantation. Transplant Proc. 2001;33(1-2):1350-1351.

5. Dew MA, Kormos RL, DiMartini AF, et al. Prevalence and risk of depression and anxiety-related disorders during the first three years after heart transplantation. Psychosomatics. 2001;42(4):300-313.

6. Kaba E, Thompson DR, Burnard P, Edwards D, Theodosopoulou E. Somebody else's heart inside me: a descriptive study of psychological problems after a heart transplantation. Issues Ment Health Nurs. 2005;26(6):611-625.

7. Lipshutz GS, Wilkinson AH. Pancreas-kidney and pancreas transplantation for the treatment of diabetes mellitus. Endocrinol Metab Clin North Am. 2007;36(4):1015-1038. 
8. Smith GC, Trauer T, Kerr PG, Chadban SJ. Prospective qualityof-life monitoring of simultaneous pancreas and kidney transplant recipients using the 36-item short form health survey. Am J Kidney Dis. 2010;55(4):698-707.

9. Sureshkumar KK, Patel BM, Markatos A, Nghiem DD, Marcus RJ. Quality of life after organ transplantation in type 1 diabetics with endstage renal disease. Clin Transplant. 2006;20(1):19-25.

10. Speight J, Reaney MD, Barnard KD. Not all roads lead to Rome-a review of quality of life measurement in adults with diabetes. Diabet Med. 2009;26(4):315-327.

11. Langenbach M, Stippel D, Beckurts KT, Geisen, Köhle K. How do patients experience their body after simultaneous pancreas-kidney transplantation? Z Psychosom Med Psychother. 2004;50(1):86-102. German.

12. Morse JM, Field, Peggy Anne. Qualitative research methods. Health professionals (2nd edition) London: Sage; 1995; chapter 2:23-26.

13. Blumer H. Symbolic Interactionism: Symbolic Interaction and Ethnographic Research. New York: State University of New York Press; 1969.

14. Fetterman DM, David M. Ethnography Step by Step. Newbury Park: Sage; 1989.

15. Miles MB, Huberman AM. Qualitative Data Analysis: An Expanded Sourcebook. Newbury Park: Sage; 1994

16. Morrow SL. Quality and trustworthiness in qualitative research in counseling psychology. Journal of Counseling Psychology. 2005;52(2): 250-260.

17. Acuña Delgado A. The body in the interpretation of cultures. Boletín Antropológico. 2001;1(51):31-52. Spanish.

18. Yang SC, Kuo PW, Wang JD, Lin MI, Su S. Quality of life and its determinants of hemodialysis patients in Taiwan measured with WHOQOLBREF(TW). Am J Kidney Dis. 2005;46(4):635-641.

19. Bradley C. Design of a renal-dependent individualized quality of life questionnaire. Adv Perit Dial. 1997;13:116-120.

20. Parkerson GR Jr, Gutman RA. Perceived mental health and disablement of primary care and end-stage renal disease patients. Int J Psychiatry Med. 1997;27(1):33-45.

21. Ifudu O, Paul HR. Effect of missed hemodialysis treatments on mortality in patients with end-stage renal disease. Nephron. 1998;79(3): 385-386.

22. Lózano M, Pandin JJ, Anasagasti JJ, et al. Liaison psychiatry in hemodialysis patients and renal transplant. Monogr Psiquiatr. 1995;7:30.
23. Pérez-San-Gregorio MA, Martín-Rodríguez A, Galán-Rodríguez A, Pérez-Bernal J. Psychologic stages in renal transplant. Transplant Proc. 2005;37(3):1449-1452.

24. Langenbach M, Kuhn-Régnier F, Geissler HJ. Patients' subjective reconstruction of meaning after heart transplantation. Transplant Proc. 2002;34(6):2183-2184.

25. Dubovsky SL, Metzner JL, Warner RB. Problems with internalization of a transplanted liver. Am J Psychiatry. 1979;136(8):1090-1091.

26. Feinstein S, Keich R, Becker-Cohen R, Rinat C, Schwartz SB, Frishberg Y. Is noncompliance among adolescent renal transplant recipients inevitable? Pediatrics. 2005;115(4):969-973.

27. Butler JA, Roderick P, Mullee M, Mason JC, Peveler RC. Frequency and impact of nonadherence to immunosuppressants after renal transplantation: a systematic review. Transplantation. 2004;77(5); 769-776.

28. Frazier PA, Tix AP, Barnett CL. The relational context of social support: Relationship satisfaction moderates the relations between enacted support and distress. Pers Soc Psychol Bull. 2003;29(9): 1133-1146.

29. Grandi S, Fabbri S, Tossani E, Mangelli L, Branzi A, Mangelli C. Psychological evaluation after cardiac transplantation: the integration of different criteria. Psychother Psychosom. 2001;70(4): 176-183.

30. Drent G, De Geest S, Dobbels F, Kleibeuker JH, Haagsma EB. Symptom experience, nonadherence and quality of life in adult liver transplant recipients. Neth J Med. 2009;67(5):161-168.

31. Silva DGV, Francioni FF, Matosinho MMS, Coelho MS, Sandoval RCB, et al. Pessoas con diabetes mellitus: suas escolhas de cuidados e tratamentos. (People with diabetes. Their choices of care and treatment.) Rev Bras enferm. 2006;59(3):297-302. Spanish.

32. Garro LC. Narrative representations of chronic illness experience: cultural models of illness, mind, and body in stories concerning the temporomandibular joint (TMJ). Soc Sci Med. 1994;38(6):775-788.

33. Ross H, Abbey S, De Luca E, Mauthner O, McKeever P, Shildrik M, Poole J. What they said vesus what we see: "hidden" distress and impaired quality on life in heart transplant recipients. J Heart Lung Transplant. 2010;29(10):1142-1149. Epub 2010 Jul 1.

34. Speight J, Sinclair AJ, Browne JL, Woodcock A, Bradley C. Assessing the impact of diabetes on the quality of life of older adults living in a care home: validation of the ADDQoL Senior*. Diabet Med. Epub July 18, 2012.
Patient Preference and Adherence

\section{Publish your work in this journal}

Patient Preference and Adherence is an international, peer-reviewed, open access journal focusing on the growing importance of patient preference and adherence throughout the therapeutic continuum. Patient satisfaction, acceptability, quality of life, compliance, persistence and their role in developing new therapeutic modalities and compounds to

\section{Dovepress}

optimize clinical outcomes for existing disease states are major areas of interest. This journal has been accepted for indexing on PubMed Central. The manuscript management system is completely online and includes a very quick and fair peer-review system. Visit http://www.dovepress.com/ testimonials.php to read real quotes from published authors. 